\title{
Subluminal Magnetic Monopole Search with NOvA
}

\author{
M. J. Frank*十 \\ University of South Alabama \\ E-mail: mfrankesouthal abama.edu
}

\author{
A. Antoshkin \\ Joint Institute for Nuclear Research \\ E-mail: antoshkinejinr.ru

\section{E. C. Dukes} \\ University of Virginia \\ E-mail: ecd3m@virginia.edu

\section{R. Ehrlich} \\ University of Virginia \\ E-mail: ehrlich@virginia.edu

\section{E. Song} \\ University of Virginia \\ E-mail: es $4 r e d v i r g i n i a . e d u$
}

Proof of the existence of the magnetic monopole has eluded physicists for centuries. The NOvA far detector (FD), used for neutrino oscillation searches, has the ability to identify magnetic monopoles at subluminal velocities $(\mathrm{v}<\mathrm{c} / 100)$. With a surface area of over $4,000 \mathrm{~m}^{2}$ and a location near the earth's surface, the $14 \mathrm{kt}$ FD provides unique sensitivity to potential low-mass monopoles unable to penetrate underground experiments. We have designed a novel data-driven triggering scheme that continuously searches the FD's live data stream for monopole-like patterns. At the offline level, the largest challenge in reconstructing monopoles is to reduce the $148,000 \mathrm{~Hz}$ speed-of-light cosmic ray background. This poster presents the trigger algorithm, the offline reconstruction algorithm, as well as the background rejection methods that will be used for the first NOvA monopole search.

36th International Cosmic Ray Conference -ICRC2019-

July 24th - August 1st, 2019

Madison, WI, U.S.A.

\footnotetext{
* Speaker.

${ }^{\dagger}$ On behalf of the NOvA Collaboration (for collaboration list see PoS(ICRC2019)1177).
} 


\section{Introduction}

\subsection{NOvA}

The NOvA experiment looks for the conversion rate of muon neutrinos into electron neutrinos. The measurement of this and the associated antineutrino rate gives information critical to understanding the mixing parameters $\theta_{23}$ and $\Delta m^{2}$, the neutrino mass hierarchy, and even the observed asymmetry of matter over antimatter in the universe. NOvA uses two highly segmented scintillation detectors with an almost fully active detection region, a near detector (ND) at Fermilab and a far detector (FD) in Ash River, MN. A beam of muon neutrinos produced at Fermilab travels through the earth to the FD. NOvA has made significant contributions to the scientific community's understanding of neutrinos since data collection commenced in 2014 [1].

\subsection{Search for Magnetic Monopoles}

The FD is a rectangular liquid scintillator detector with a mass of over $14 \mathrm{kt}$ and a size of $15 \mathrm{~m}$ by $15 \mathrm{~m}$ by $60 \mathrm{~m}$, it is constructed of 896 planes that together contain a total of 344,064 channels (cells). Thanks to the data-driven trigger (DDT) system, the FD is able to isolate interesting physics signals hidden among the 148,000 cosmic rays crossing it every second. The DDT system allows placement of the FD near the surface of the earth under only modest shielding. Such a sensitive and large detector has never been placed in such an exposed location before, which opens up a previously inaccessible phase space to search for subluminal $\left(\beta<10^{-2}\right)$ exotic particles that would usually be absorbed by large shielding or overburdens of other experiments. One such exotic particle is the magnetic monopole. Though posited by widely-credited theoretical work [2], searches over the past century have yet to find evidence of such a particle's existence.

This analysis is the first to probe uncharted territory for subluminal, intermediate mass magnetic monopoles (Figure 1), but it is only the first in a series of analyses spanning the entire dataset to be collected by NOvA's full physics run. At the end of NOvA's run, our search sensitivity can be competitive with the leading magnetic monopole searches to date (e.g. MACRO).

This document describes the algorithm designed to search the FD data for slowly moving particles. The event topology is therefore a straight track traversing the FD with a velocity that is a fraction of the speed of light. The signal events were simulated using subluminal magnetic monopoles. As usual, $\beta$ is defined as the ratio between the particle's velocity $(v)$ and the speed of light $(c)$. This search has been optimized for $\beta=10^{-3}$ magnetic monopoles, but has sensitivity to monopoles as slow as $\beta=3 \times 10^{-4}$ and as fast as $5 \times 10^{-3}$. Another dedicated search for fast monopoles has optimized its analysis strategy for monopoles faster than this [3].

\section{Magnetic Monopole Simulation}

\subsection{Energy Deposition Model}

The magnetic monopole search identifies highly-ionizing particles traversing the FD at velocities slower than the speed of light. Because the monopole energy deposition approaches minimum ionizing energy deposition for slow magnetic monopoles $\left(\beta \sim 10^{-4}\right)$, the slow monopole search uses timing techniques for identification. 


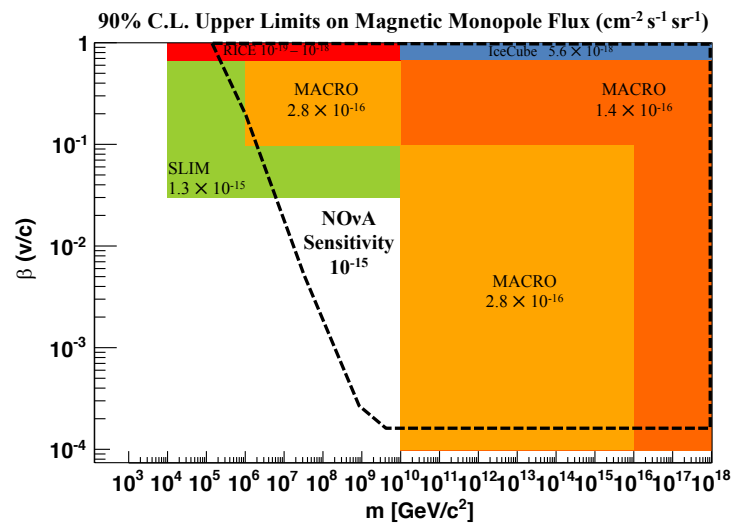

Figure 1: This graphic shows the full range of velocity/mass space where the magnetic monopole could exist. The colored boxes correspond to searches performed by the MACRO, SLIM, RICE, and IceCube collaborations $[4,5,6,7]$. The area enclosed by the dashed line shows NOvA's approximate sensitivity after six years of running. The white region within the dashed line represents the region that no other experiment has been able to access.

Ahlen and Kinoshita calculated the energy deposition model for slow monopoles $\left(\beta<10^{-2}\right)[8]$, which is given by:

$$
\frac{d E}{d x}=a N_{e}^{\frac{2}{3}}\left[\ln \left(b N_{e}^{\frac{1}{3}}\right)-\frac{1}{2}\right] \beta
$$

where $N_{e}$ is the electron density, which depends on the material. The two other constants ( $a$ and $b$ ) can be expressed in terms of the following material-independent constants:

$$
a=\frac{2 \pi g^{2} e^{2}}{\hbar c\left(3 \pi^{2}\right)^{1 / 3}} \quad b=2\left(3 \pi^{2}\right)^{1 / 3} a_{0}
$$

where $e$ is the fundamental charge, $\hbar$ is the reduced Planck constant, $c$ is the speed of light, and $a_{0}$ is the Bohr radius. $g$ stands for the monopole charge and it is set to the Dirac charge for this analysis:

$$
g=g_{\text {Dirac }}=\frac{e}{2 \alpha}=\frac{\hbar c}{2 e}
$$

where $\alpha$ is the fine structure constant.

A monopole traversing the FD deposits visible energy in the liquid scintillator. NOvA's liquid scintillator is mostly comprised of mineral oil (solvent) and pseudo-cumene (scintillant), and its electron density is given by [3]:

$$
N_{e}(\text { Scintillator })=2.9 \times 10^{23} \mathrm{~cm}^{-3}
$$

Using Eq. 2.1, this yields the following slow monopole energy deposition equation in NOvA's liquid scintillator:

$$
\frac{d E}{d x}=\left(12 \frac{\mathrm{GeV}}{\mathrm{cm}}\right) \beta
$$


This energy deposition model is used for the monopole velocity range of $10^{-4}<\beta<10^{-2}$.

\subsection{Monte Carlo (MC) Simulation}

Geant 4 is used to simulate the monopole. In the absence of information about the distribution of the direction of the monopoles, the probability is assumed to be identical for monopoles coming from any direction, so an isotropic distribution of the monopole directions is used in the simulation. The surface entry point is chosen at random, and the simulated monopole is given direction and speed parameters $(\beta)$. The speed determines the energy deposition rate (Eq. 2.5). The monopole energy is very large relative to the energy lost in the detector, so it is assumed that the monopole's velocity vector remains constant.

\section{Samples}

This analysis uses two sets of data samples. One characterizes monopole signal events, and the other is the data sample to be analyzed for slowly moving particles.

\subsection{Signal MC Sample}

Monopoles at various velocities are simulated and propagated through the FD, with one monopole per simulated event. Next, each simulated event is combined with a $5 \mathrm{~ms}$ long min-bias data produced by a daily trigger. This results in an overlayed event that contains both the true monopole and nominal detector activity over a $5 \mathrm{~ms}$ time period. This MC sample is used to measure how well the search algorithm can identify slow monopoles and differentiate them from the overwhelming cosmic ray background present in $5 \mathrm{~ms}$ of data.

\subsection{Background Data Sample}

The data is produced by the slow monopole trigger that operates as part of NOvA's DataDriven Trigger (DDT) system [9]. The version of the slow monopole trigger used here was deployed on June $5^{\text {th }}, 2015$, which defines the beginning of the data sample. On October $12^{\text {th }}, 2015$, the FD was reconfigured to a higher gain setting, which is used as the end of this data sample. The training data shown here corresponds to $10 \%$ of the full data sample and is labeled min-bias data. The assumption is that no monopole exists in this subset of the data. Once the requirements on the reconstruction parameters are established using this $10 \%$ background data sample, the remainder of the triggered data is unblinded and searched for monopole-like events.

\section{Online Trigger Algorithm}

The slow monopole trigger algorithm, initially inspired by IceCube's Slow-Particle-Trigger [10], attempts to identify straight lines of hits consistent with a slow track. One event in the DDT world corresponds to a $5 \mathrm{~ms}$ readout window. The trigger algorithm starts by considering pairs of hits on the surface of the detector for one such 5-ms-event. Since time performance is one of the most important concerns for the live trigger, the algorithm needs to make a decision as quickly as possible. To that end, it identifies hits that lie on a 20 cell wide "road" between the original surface hits (see Figure 2). The algorithm then looks for gaps between the hits on the road and identifies 
the maximum plane gap (along the $z$-axis) and the maximum cell gap (along the $x$ - or $y$-axes). If there is a plane gap larger than 30 planes or a cell gap larger than 20 cells, the algorithm rejects the pair of hits. The procedure is then repeated for all of the pairs of hits on the detector surface. When this trigger algorithm was deployed, the trigger budget lacked the necessary time to consider all possible pairs of surface hits, so only every tenth pair is checked. This sparsification method is efficient for monopole signal because a true monopole signal would have many pairs of surface hits. The pairs of surface hits must also have sufficient separation in space and time to originate from a track with a velocity $(\beta)$ of less than $5 \times 10^{-3}$. If the algorithm identifies a pair of surface hits without large gaps, the event is written out to permanent storage.

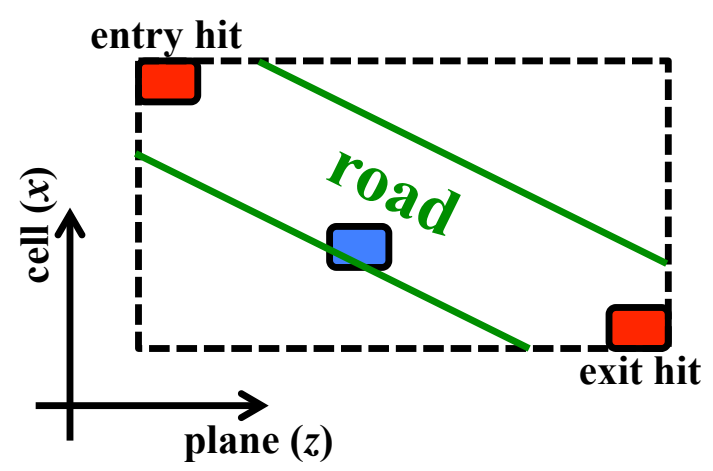

Figure 2: Schematic depiction of the trigger algorithm. The red hits are on the detector surface and are labeled entry and exit based on their time. The algorithm looks for large gaps between hits on the road. A large gap causes the pair to be rejected. Otherwise, the slow monopole-like event is written out.

\section{Offline Reconstruction Algorithm}

The FD read-out electronics acquire data in two projected views separately, the $x z$ - and $y z$ views. The $y$-direction is the vertical dimension while the $z$-direction points along the neutrino beam originating from Fermilab. The algorithm starts by extracting two-dimensional information from each view and then combines this information into three-dimensional objects known as monopole tracks.

Figure 3 illustrates how the analysis algorithm is able to pick simulated monopole events out of the formidable background. First, the algorithm identifies all of the fast moving cosmic ray muons and removes them from the event. The algorithm then removes isolated as well as uncorrelated activity. The remaining energy depositions (hits) are reconstructed using the Hough Tracking Algorithm to identify straight line objects. A line is fitted to the collection of hits associated with the monopole track. Next, the linear correlation coefficient (Sec. 5.1), time gap fraction (Sec. 5.2), and velocity $(\beta)$ are calculated for this monopole track and used to select monopole signal events (Sec. 5.3). At a minimum, a monopole track must meet the following three requirements:

1. There must be at least twenty hits in each projection ( $x z$ and $y z)$.

2. The track must cross at least ten planes in each projection ( $x z$ and $y z)$. 
3. The track must have a reconstructed length of at least $10 \mathrm{~m}$.
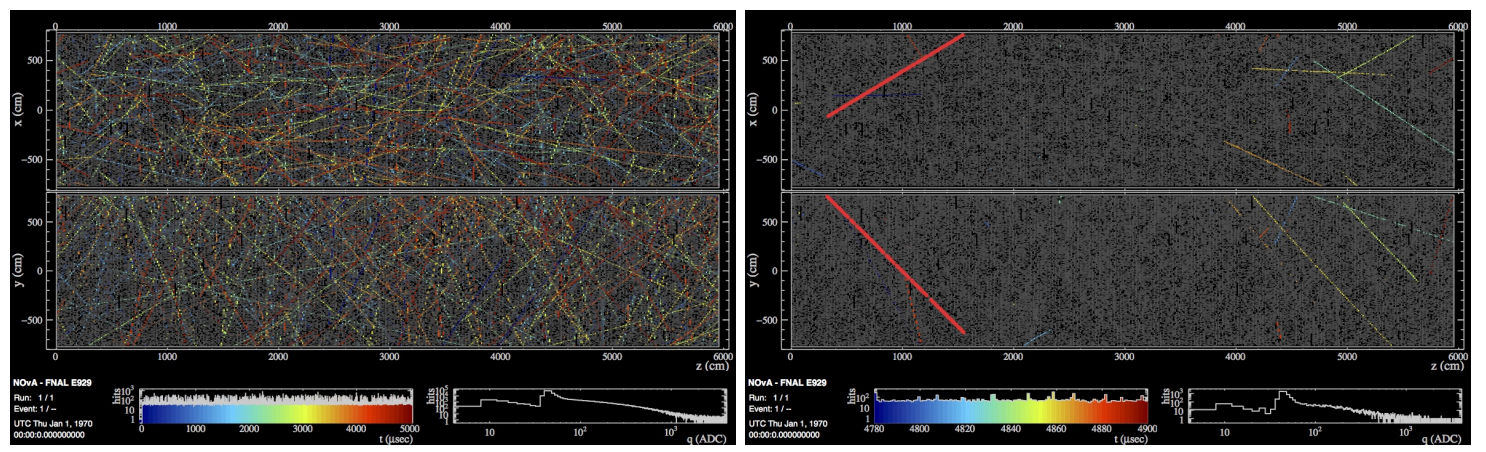

Figure 3: Two NOvA event displays of raw data with a simulated magnetic monopole superimposed. The event displays show the two projected views ( $x z$ and $y z$ ) of the FD. The left event display shows the overwhelming cosmic ray background in $5 \mathrm{~ms}$ of data that needs to be suppressed to reveal the slow-moving magnetic monopole. The right event display then shows the result of the reconstruction algorithm properly identifying the simulated monopole. The right-hand event display shows a smaller time window of $120 \mu \mathrm{s}$ to highlight the simulated magnetic monopole marked in red.

\subsection{Linear Correlation}

Once a line is fitted to the collection of hits associated with the monopole track, calculation using standard linear regression techniques yields the squared correlation coefficient $\left(r^{2}\right)$ for hits in the $x t$ - and $y t$-spaces separately. A value of $r^{2}$ equal to unity means that the hits lie on a straight line while a value of zero means that the hits are uncorrelated [11]. For a high-quality track, the linear correlation coefficient must be close to unity. This calculation is performed separately for each view, yielding $r_{x t}^{2}$ and $r_{y t}^{2}$ for each track. The minimum of these two quantities is called $r_{\min }^{2}$. Therefore, if $r_{\min }^{2}$ must have a large value close to unity, both $r_{x t}^{2}$ and $r_{y t}^{2}$ will have to exceed this requirement. Figure 4 (left) shows the strength of $r^{2}$ in separating signal (MC: red) from background (data: black).

\subsection{Time Gap Fraction}

For the analysis of the hits to be meaningful, they should also follow a temporal progression. Many of the reconstruction failures occur when two independent speed-of-light cosmic rays are identified as a single monopole track. Such monopole tracks have a cluster of hits occurring early in time, a large time gap, and then another cluster of hits occurring later. To exclude this reconstruction failure mode, the largest time gap between consecutive hits must meet certain requirements.

First, the hits associated with the monopole track are sorted by time, and the largest gap in time between adjacent hits $\left(\Delta t_{\max }\right)$ is calculated. The time gap fraction is then defined by:

$$
f=\frac{\Delta t_{\max }}{\Delta t_{\text {track }}}
$$

where $\Delta t_{\text {track }}$ is the difference in time between the first and last hits of the monopole track sorted by time. A high-quality track will have a value of $f$ close to zero indicating that there are no gaps 
in time along the track. A track that consists of two independent clusters of hits as described above will have a value of $f$ close to unity. The algorithm therefore requires $f$ to be close to zero. This calculation is performed separately for each view, yielding $f_{x t}$ and $f_{y t}$ for each track. The maximum of these two quantities is called $f_{\max }$. If $f_{\max }$ must have a small value close to zero, both $f_{x t}$ and $f_{y t}$ have to be below this requirement. Figure 4 (right) shows the strength of $f$ in separating signal (MC: red) from background (data: black).
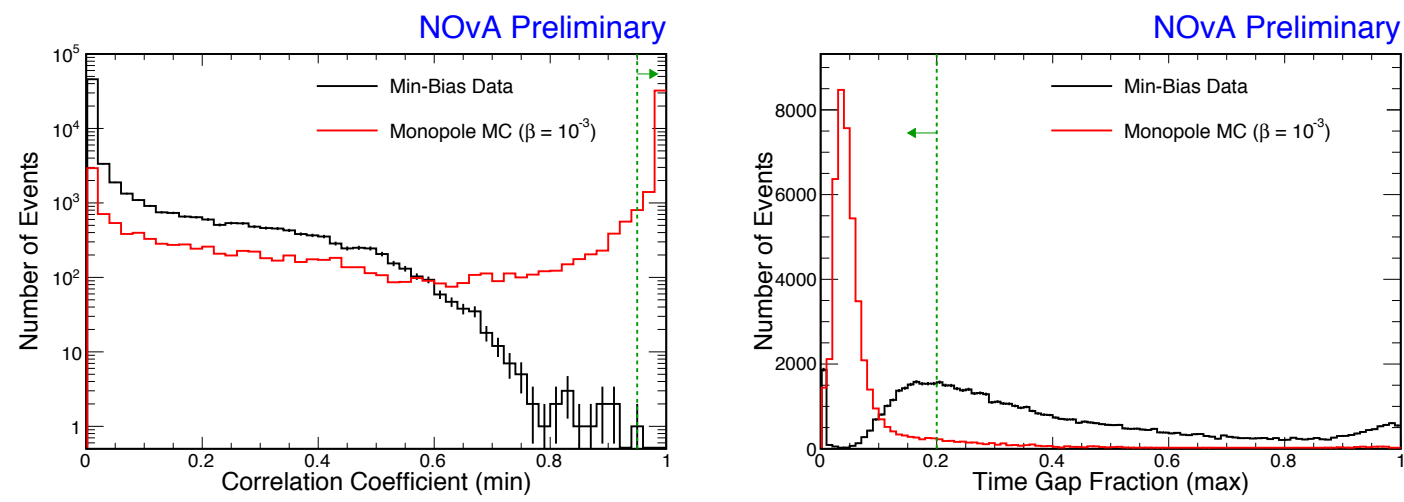

Figure 4: The histograms show the reconstructed correlation coefficient $r_{\text {min }}^{2}$ (left) and the time gap fraction $f_{\max }$ (right) of the primary monopole track for data (black) and MC (red). The ordinate axes show the number of events in each bin. Please note that the cosmic rays have already been removed from the min-bias data, so the linearity measures are weak.

\subsection{Monopole Signal Selection Requirements}

Training the algorithm on the NOvA data began with the assumption that no monopole existed in small subsamples of the full data set (Sec. 3.2). This was first done with samples approximately $0.3 \%, 1 \%$ and then $10 \%$ of the full sample. The signal selection requirements arrived at with the $10 \%$ sample are indicated by the dashed green lines in Figure 4 and are given by:

1. $f_{\max } \leq 0.2$

2. $r_{\min }^{2} \geq 0.95$

3. $\beta<10^{-2}$

Figure 5 shows the final distribution of simulated signal (red) and data (black). The figure caption explains the details, but the strength in the reconstruction algorithm is clearly visible by the lack of background events in the signal region. The reconstruction efficiency for the simulated $\beta=10^{-3}$ monopole sample is $72 \%$.

\section{Conclusion}

The search strategy for magnetic monopoles in the NOvA FD has been presented here. The search over the full data sample is currently under collaboration-wide review and will be presented soon. 


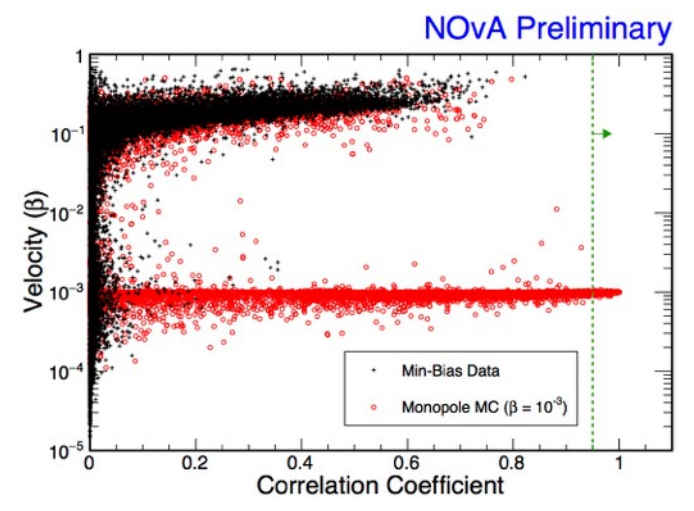

Figure 5: This scatter plot shows the reconstructed monopole velocity $(\beta)$ and correlation coefficient $\left(r^{2}\right)$ for the min-bias data sample (black) and the simulated signal sample (red). The signal cut on the correlation coefficient is indicated by the green dashed line. A signal monopole event would have to be slow $\left(\beta<10^{-2}\right)$. Here, the signal sample densely populates the signal region ( $72 \%$ of signal events), while none of the minbias data enters this region. Note that the low time gap requirement $\left(f_{\max } \leq 0.2\right)$ has already been applied to these events.

\section{Acknowledgments}

This document was prepared by the NOvA collaboration using the resources of the Fermi National Accelerator Laboratory (Fermilab), a U.S. Department of Energy, Office of Science, HEP User Facility. Fermilab is managed by Fermi Research Alliance, LLC (FRA), acting under Contract No. DE-AC02-07CH11359. This work was supported by the U.S. Department of Energy; the U.S. National Science Foundation; the Department of Science and Technology, India; the European Research Council; the MSMT CR, GA UK, Czech Republic; the RAS, RFBR, RMES, RSF, and BASIS Foundation, Russia; CNPq and FAPEG, Brazil; STFC, and the Royal Society, United Kingdom; and the state and University of Minnesota. This work used resources of the National Energy Research Scientific Computing Center (NERSC), a U.S. Department of Energy Office of Science User Facility operated under Contract No. DE-AC02-05CH11231. We are grateful for the contributions of the staffs of the University of Minnesota at the Ash River Laboratory and of Fermilab.

\section{References}

[1] M. A. Acero et al. (NOvA Collaboration), Phys. Rev. D 98032012 (2018).

[2] M. Tanabashi et al. (Particle Data Group), Phys. Rev. D 98030001 (2018).

[3] Z. Wang, Ph.D. thesis, University of Virginia (2015).

[4] M. Ambrosio et al. (MACRO Collaboration), Eur. Phys. J. C 25511 (2002).

[5] S. Balestra et al. (SLIM Collaboration), Eur. Phys. J. C 5557 (2008).

[6] D.P. Hogan et al. (RICE Collaboration), Phys. Rev. D 78075031 (2008).

[7] R. Abbasi et al. (IceCube Collaboration), Phys. Rev. D 87022001 (2013).

[8] S. P. Ahlen and K. Kinoshita, Phys. Rev. D 262347 (1982).

[9] A. Norman et al., J. Phys. Conf. Ser. 664082041 (2015).

[10] M. G. Aartsen et al. (IceCube Collaboration), Eur. Phys. J. C 742938 (2014).

[11] D. A. McQuarrie, Mathematical Methods for Scientists and Engineers, University Science Books (2003). 